\title{
SURFACE APPEARANCE OF TOOTH CAVITY WALLS WHEN SHAPED WITH VARIOUS INSTRUMENTS
}

\author{
F. A. PFYTON, D.Sc., AND J. F. MORTELI, JR., D.D.S. \\ School of Dentistry, Dniversity of Michigan, Ann Arbor, Mich.
}

SINCE the introduction of faster operating speeds for rotating instruments, $\checkmark$ there has been an increased concern regarding the effects of various instruments on the cut tooth surface. Very little information on this subject exists in the literature. The work of Street ${ }^{1}$ describing the effects of instruments on enamel walls represents the most complete report in the current literature.

It is the purpose of this report to describe an altemate procedure for examining the tooth surface as it exists after being cut with various instruments. Particular emphasis will be placed upon the technic and procedure of observing the eut tooth surface.

\section{ROTATING INSTRUMENTS}

It has seemed desirable not only to determine the appearance of the freshly cut tooth surface but also to examine the surface appearance of the cutting instrument. Fig. 1 shows the surface appearance of 6 inverted cone type diamond instruments. Some difference in surface texture and appearance of these instruments can be detected. Some have relatively sharp corners while others are rounded, and some display quite uneven applications of the diamond particles while others appear to have a more uniform surface texture. In Fig. 2 is shown a group of cylindrical or taper type diamond instruments. These also show a lack of uniformity in surface appearance and details of the instrument. The small disks represented in Fig. 3 show a lack of uniformity with some variation in surface roughness. The disks in Fig. 4 represent the large diameter disks suitable for the slice type of preparation, and show some evidence of different particle size and surface texture of the cutting instruments. Interesting comparisons might be made with carborundum stones and various types of dental burs, but space does not permit inclusion of all the rotating instruments in this presentation.

\section{TECHNIC FOR SAMPLE PREPARATION}

Because of the translucent nature of tooth tissue, it is difficult to examine microscopically and to record photographically the surface appearance of the freshly cut tooth by means of reflected illumination. Street used lamp black paste which was wiped on the cut surface and, after drying, the excess material was removed leaving the scratches from the instrument filled with the lamp black.

This study supported by a contract between the United States Air Force, School of Aviation Medicine, Project No. AF $16(600) 427$, and the University of Michigan, School of Dentistry.

Presented at the Annual Meeting of the Dental Materials Group of the International Association for Dental Research, French Lick Springs, Ind., March, 1954 (J. D. Res. 33: 699, 1954).

Received for publication Feb. 2, 1955 
In order to render the tooth more opaque in this study the tooth surface has been shadowed with a thin deposit of copper in the same way that shadowing is used to develop the detail of the replica technic for high power light microscope and electron microscope studies. The procedure of proper deposition was identical to that ordinarily employed for the replica technic in which a copper filament is vaporized in vacuum permitting the copper to be deposited on the surface of the specimen. The illustrations which are used in this report represent teeth that were placed in the vacuum chamber and had the copper deposited directly on them rather than on a replica of the tooth surface. The technical details of the operation of shadow deposition have been adequately described in the literature. ${ }^{2}$ The thickness of the metal deposit can be controlled by the time and condition of deposition. In these studies, the deposit was not sufficiently thick to alter the surface appearance resulting from the action of the cutting instrument, but was suffieient to render the surface somewhat opaque and permit better microscopic examination.

Fig. 1.

Fig. 2.
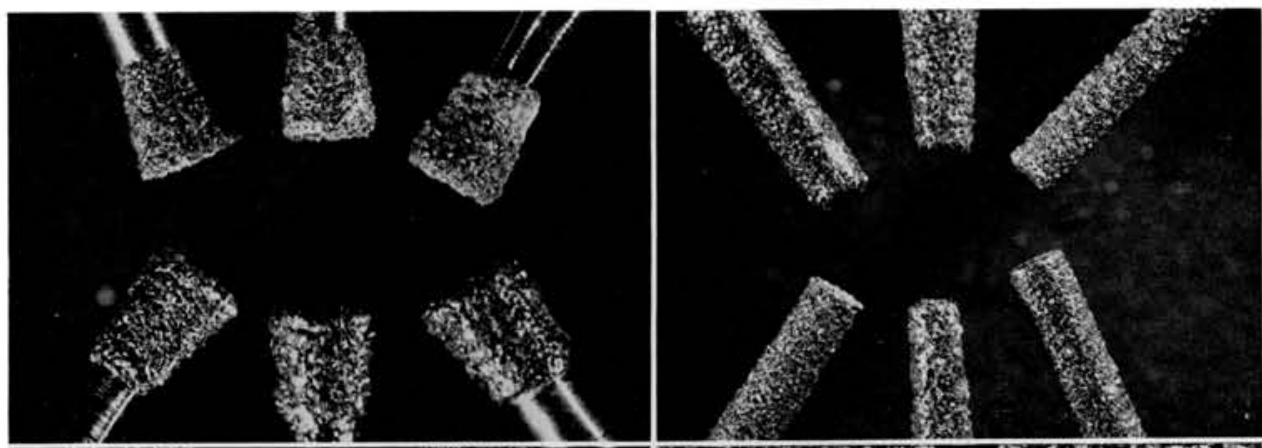

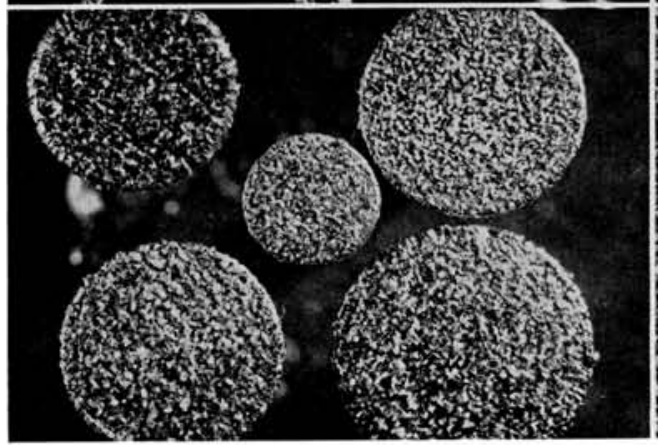

Fig. 3 .

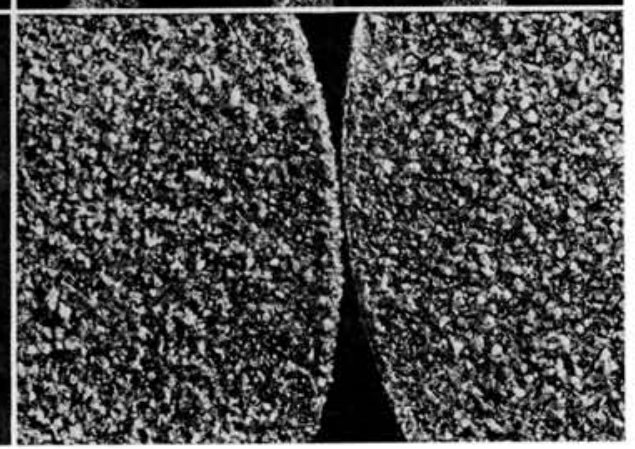

Fig. 4.

Fig. 1.-Representative inverted cone diamond instruments.

Fig. 2.-Cylindrical and taper type diamond instruments.

Fig. 3.-Variations in surface texture of small diamond disks.

Fig. 4.-Two representative large size diamond disks.

Freshly extracted teeth were chosen and were kept wet until the eavity was shaped with the various instruments chosen. Studies were made using a variety of diamond instruments at both high (12,000 r.p.m.) and low $(4,000$ 
r.p.m.) speeds of operation. A variety of carborundum disks were used at both high and low speeds in the dry condition while diamonds were operated both wet and dry. The surface developed by the use of an enamel hatchet has been included. Photographs of the cutting instruments and the surfaces developed on the cut tooth have been recorded at relatively low magnifications of approximately 20 diameters. Some details seem to be lost because of lack of depth of focus when higher magnifications are employed and lower magnifications do not reveal the full surface appearance.

\section{SEPARATING DISKS}

An interesting surface results from the application of a "Joe Dandy" type of disk to slice the tooth as shown in Fig. 5. Such a disk operated dry at slow speed develops a series of symmetrical seratches, some of which appear to be much deeper than others. While the surface appears to be uniformly abraded, it cannot be interpreted as representing a smooth surface. In Fig. 6 is shown the surface developed by the same disk, when operated wet and at fast speeds. While this illustration represents a contour cut of the tooth rather than a straight slice, the cut surfaces are similar in appearance with respect to the disk action on the tooth. There is no pronounced evidence of a different surface appearance resulting from the same instrument at different speeds, or when used wet or dry. The surface developed by a so-called "lightning disk" is shown by Fig. 7. This represents a slice comparable to that of Fig. 5. The disk was operated dry at a slow speed for this eut. While the cut resembles closely that made by the "Joe Dandy" disk in general appearance, there is perhaps a less pronounced and less well-defined scratch developed by this disk. Possibly this is related to the different texture of the disk.

\section{DIAMOND DISKS}

In general, it appears that the surfaces resulting from the action of diamond disks have scratches that are a little coarser than those from corresponding carborundum disks (Fig. 8). This represents the surface cut with a popular diamond disk operated wet and at high speeds. It is apparent that the operator changed the position of the instrument during the eut but it reveals also that coarse particles on the disk left deep scratches. The surface left by the action of another product when operated wet at slow speeds is shown in Fig. 9. This surface is comparable to that of Fig. 8, and shows no significant result of the slower speed of operation.

Some dentists claim it is desirable to refinish the surface resulting from the diamond disk with a small diameter, cylindrical, or tapered carborundum stone. Such an operation is described as "honing" the surface. In Fig. 10 is shown a portion of a tooth which was first sliced with a diamond disk, after which the portion on the right was finished with a No. 28 pointed white stone of small diameter. The surface appearance of the two areas is quite different with the surface scratches less pronounced where the stone was used, although this surface presents a lack of uniformity due to the uneven action of the small diameter stone. 
Fig. 5 .

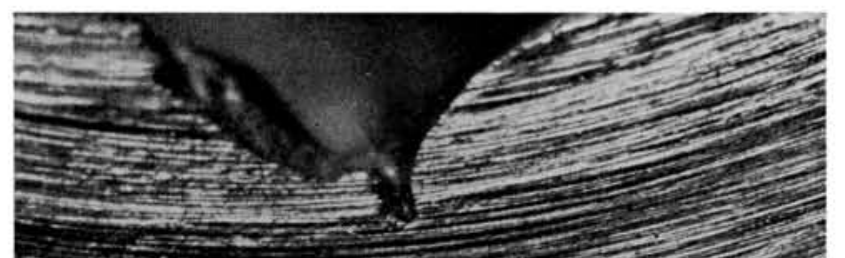

Fig. 6.
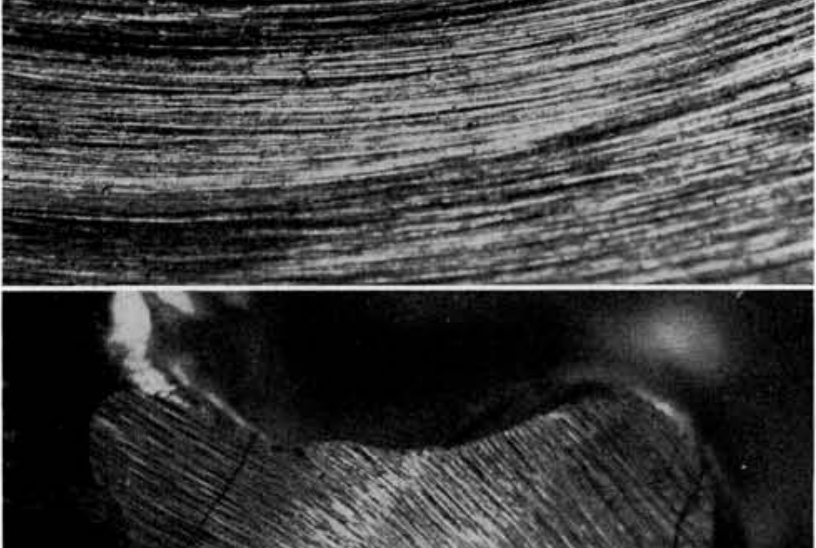

Fig. 7 .

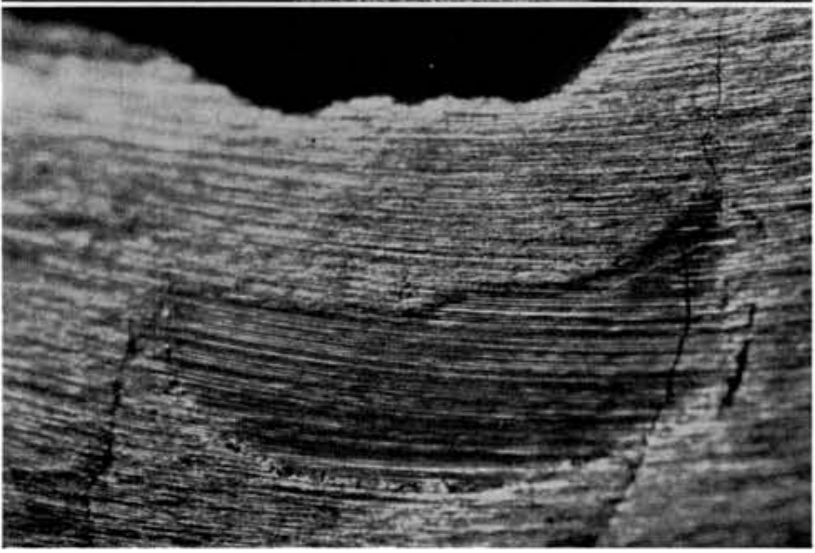

Fig. 5.-Tooth surface left from action of "Joe Dandy" disk operated slowly and dry. Fig. 6.- Result of cutting tooth with "Joe Dandy" disk while wet at fast speed. Fig. 7.-Cut surface resulting from use of lightning disk dry at slow speeds. 


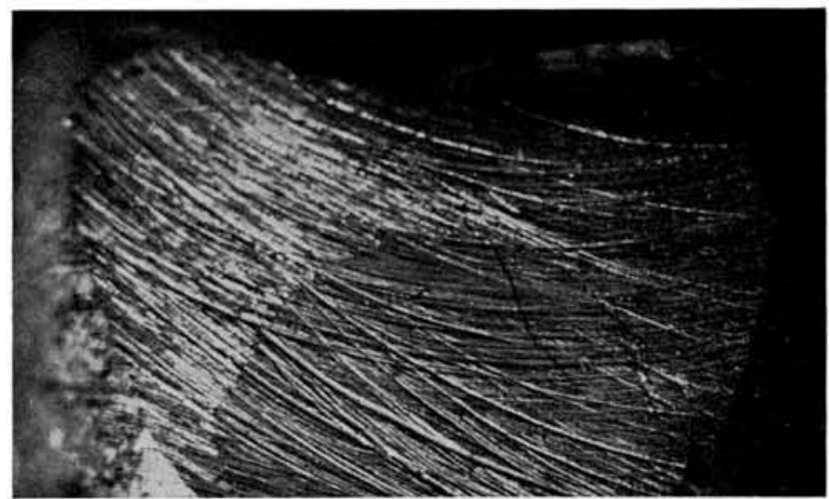

Fig. 8.

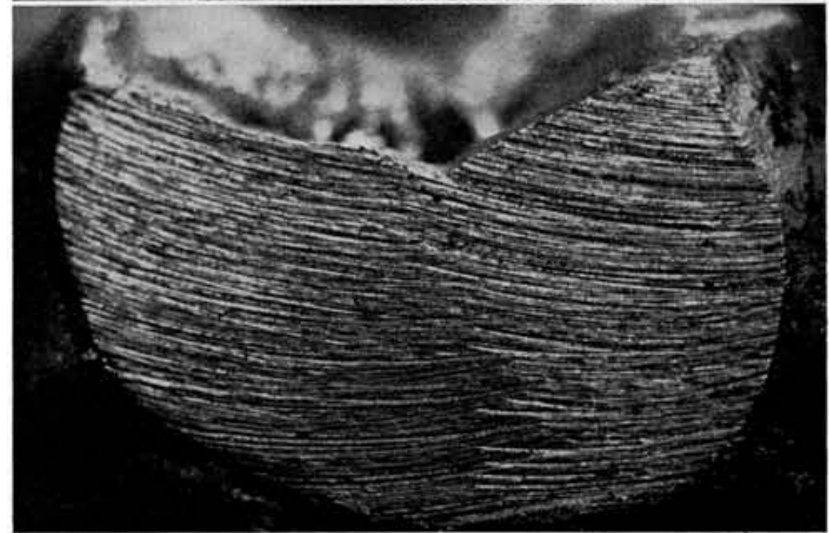

Fig. 9.

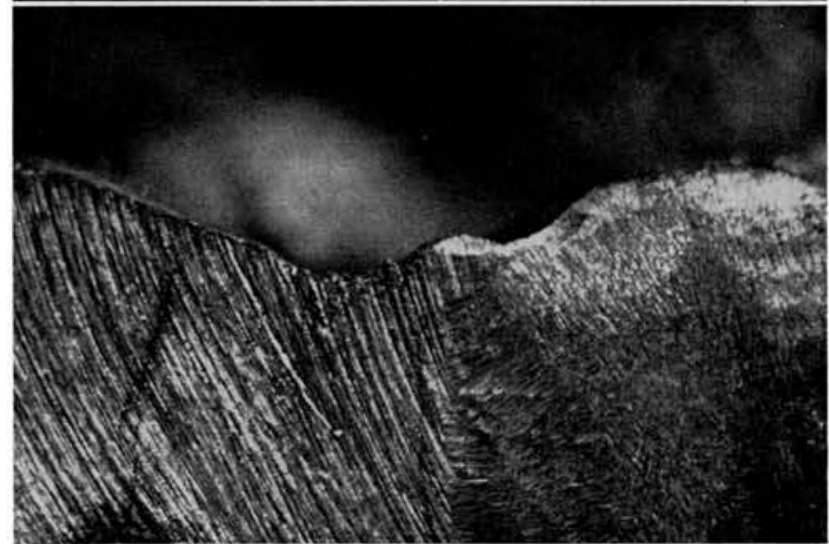

Fig. 10.

Fig. 8.- Surface produced by diamond disk operated wet at high speeds.

Fig. 9.- Surface produced by diamond disk operated wet at slow speeds.

Fig. 10,-Surface resulting from application of diamond disk at left and carborundum stone at right. 


\section{NO. 560 DIAMONDS}

In the shaping of certain cavities it is often desirable to employ a straight fissure type diamond instrument No. 560, of a small diameter. In size and shape this instrument resembles the No. 59 straight fissure bur. When applied dry at slow speeds to the tooth structure, there results a surface appearance which lacks uniformity both with respect to the depth of the surface seratehes and also from one area of the tooth to another.

Fig. 11.
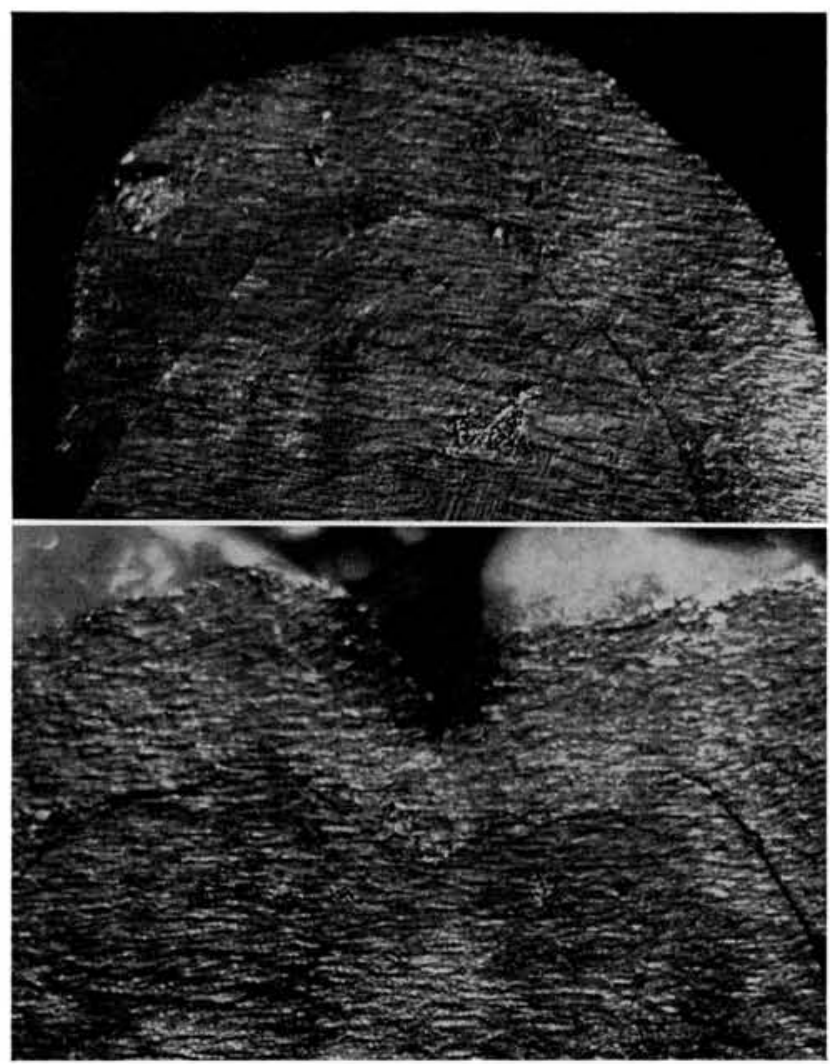

Fig. 12.

Fig. 11.-Appearance of tooth after being cut with No. 560 type diamond when operated wet at slow speeds.

Fig. 12.-Appearance of tooth after being cut with No. 560 diamond when operated wet at high speeds.

When using the same instrument wet at slow speeds the resulting tooth surface is shown in Fig. 11. This surface resembles closely those resulting from using the instrument dry and also shows the effect of the irregular diamond particles in the instrument. In Fig. 12 is the surface developed from this same instrument operated wet at high speeds. From these observations there seems to be no evidence to indicate that a different tooth surface results from either 
different operating speeds or with the presence or absence of water during the cutting operation. While the surface from the small diameter diamond resembles in some ways that of a small diameter carborundum stone, it is generally filled with deeper scratches from a diamond instrument than from a similar carborundum instrument.

\section{DENTAL BURS}

By contrast with the diamond and carborundum instruments the surface resulting from the action on the tooth of a No. 558 steel cross-cut fissure bur when operated dry at slow speeds, is shown in Fig. 13. The surface presents

Fig. 13.

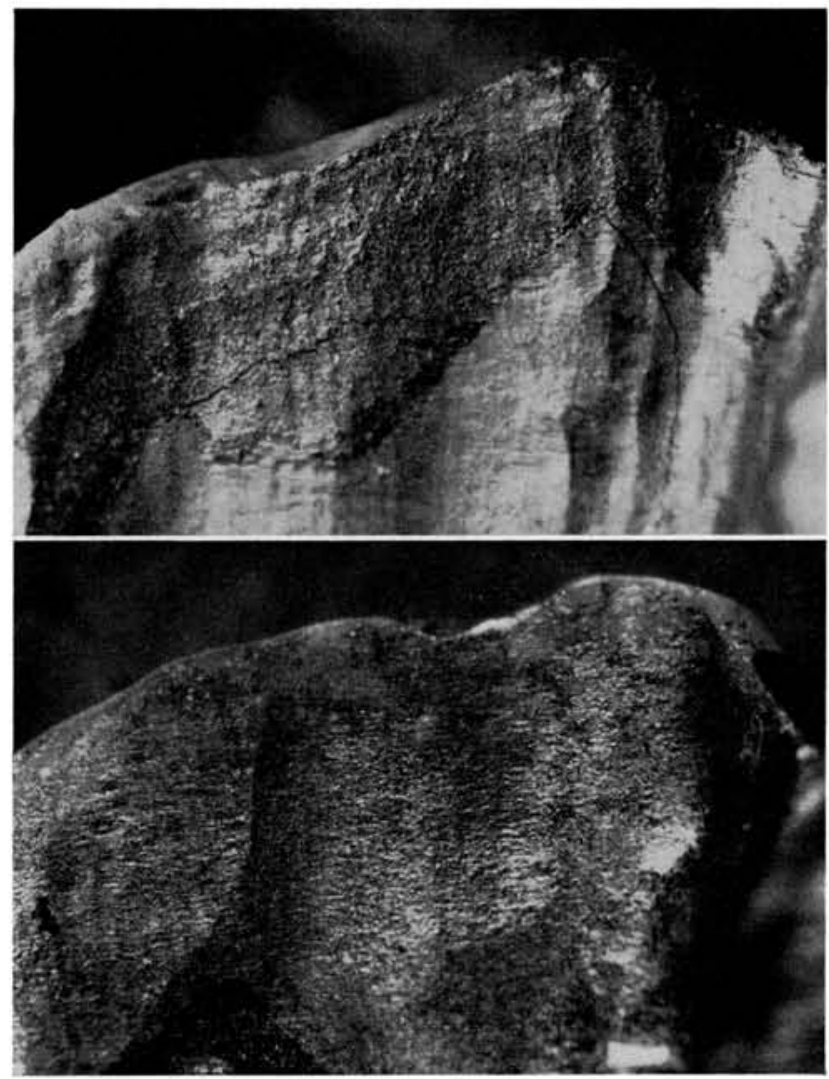

Fig. 14 .

Fig. 13.-Relatively smooth surface of tooth resulting from action of No. 558 steel crosscut fissure bur.

Fig. 14.-Surface of cut tooth resulting from action No. 58 straight fissure bur.

less evidence of deep seratches than with the diamond but shows a nonuniformity due to the uneven cutting action from one area to another. No significant differences could be detected when the bur was operated fast or slow, or when wet or dry. The surface resulting from a No. 702 cross-cut taper fissure bur is essentially the same as that from the No. 558 bur. 
When using a No. 58 steel straight fissure bur dry at low speeds, the surface developed is similar to that shown in Fig. 14. This surface represents perhaps the smoothest and most uniform one observed with small diameter rotating instruments. A similar surface results from a No. 601 steel bur which is similar in design except for a taper shape. The surface here resembles that from a planing instrument and lacks the deep seratches characteristic of diamonds and stones. Again, a change in the speed of operation did not change the surface appearance developed on the tooth by the bur.

Fig. 15.
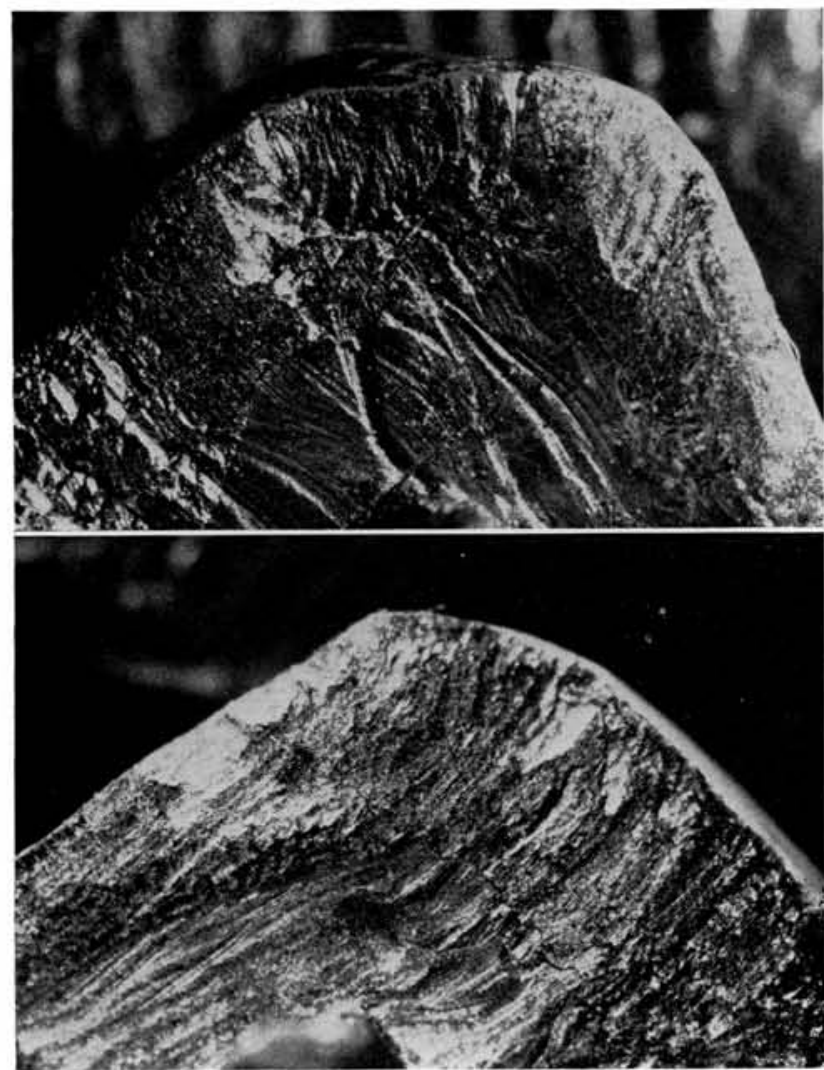

Fig. 16 .

Fig. 15.-Cavity wall resulting from cut by enamel hatchet.

Fig. 16.-Appearance of cavity wall shaped with enamel hatchet.

\section{HAND INSTRUMENTS-ENAMEL HATCHET}

Some operators have expressed the opinion that the hand instrument develops the best or smoothest surface on the eavity wall. In Fig. 15 is shown the surface resulting from the use of an enamel hatehet. This shows a lack of uniformity over the area and perhaps illustrates also that the skill of the operator in the use of the instrument is an important factor in developing a good sur- 
face in the cavity. In Fig. 16 is shown another cavity wall shaped with the same instrument. While some improvement is shown in the surface condition of the cavity wall, it is less smooth than that resulting from a fissure bur. The accessibility of the cavity to the application of the instrument may modify to some degree the results of different operators, but in general the hand instruments do not seem to present a superior surface to that resulting from rotary instruments. This agrees in general with the observations of Street.

\section{SUMMARY}

A method suitable for examining the cavity walls of the extracted tooth has been presented. This method consists of shadowing the tooth surface with a thin film of copper or other metal to make the tooth less translucent. This operation permits the examination of the cut surface by reflected light at various magnifications.

Results are presented from the use of different cutting instruments to cut tooth tissue. Little difference in results can be observed from different speeds of rotation, or from using the instrument wet or dry, when the cut surface is examined macroscopically. A difference between burs, carborundum stones, and various diamond instruments is evident.

\section{REFERENCES}

1. Street, E. V.: Effects of Various Instruments on Enamel Walls, J. A. D. A. 46: 274, 1953.

2. Williams, R. C., and Wyckoff, R. W. G.: Applications of Metallic Shadow Casting to Microscopy, J. Applied Physics 17: 23, 1946. 